\title{
The VED Meter - a New Tool to Measure the Ventricular Conduction Abnormalities in Heart Failure Patients
}

\author{
Filip Plesinger ${ }^{1}$, Pavel Jurak ${ }^{1}$, Josef Halamek ${ }^{1}$, Pavel Leinveber ${ }^{1,2}$, Scott McNitt ${ }^{3}$, Arthur J. Moss ${ }^{3}$, \\ Wojciech Zareba ${ }^{3}$, Jean Philippe Couderc ${ }^{3}$ \\ ${ }^{1}$ Institute of Scientific Instruments of the Czech Academy of Sciences, Brno, Czech Republic \\ ${ }^{2}$ ICRC, St. Anne's University Hospital Brno, Czech Republic \\ ${ }^{3}$ Heart Research Follow-up Program, University of Rochester, Rochester, NY, USA
}

\begin{abstract}
Heart failure patients with a left ventricular ejection fraction below or equal to 35\%, QRS duration above 150 ms, and left bundle branch block (LBBB) are usually recommended for treatment with a cardiac resynchronization therapy (CRT) device. Yet, about onethird of these patients do not respond to CRT revealing the limit of the current patient management guidelines. For this reason, new methods for assessing the level of electrical ventricular delay (VED) are being explored.

In this work, we present the VED Meter, autonomous software measuring the level of electrical activation delay from the standard body-surface electrocardiogram (ECG). The software delivers the VED parameter expressed in ms that reflects the electrical dyssynchrony between the right ventricle, septum and left ventricle lateral wall. We applied this software to the data from the MADIT-CRT trial to evaluate the VED values in three types of ventricular conduction disturbances: $L B B B$, right bundle branch block (RBBB), and intra-ventricular conduction disturbances (IVCD). We report that VED parameter has negative values for RBBB (delayed activation of the septum and the right ventricle) and positive for $\angle B B B$ (delayed activation of the left ventricle lateral wall) which were significantly different at $p<0.001$.
\end{abstract}

\section{Introduction}

Heart failure patients with LBBB and prolonged QRS complex have a delayed electrical activation of the left ventricle lateral wall which can be reduced by the utilization of cardiac resynchronization therapy (CRT). However, one-third of CRT recipients currently prescribed with a CRT device do not respond to the therapy [1], [2]. For this reason, we proposed a method for the measurement of the ventricular electrical dyssynchrony [3], [4] from high-resolution ECG recordings (analysing frequency range $500-1000 \mathrm{~Hz}$ ). The method was modified to work with ECG sampled at $1 \mathrm{kHz}$ and implemented within software.
We present this software called VED Meter (C) ISICAS, Brno, Czech Republic, version 1.3.3) and its application to the MADIT-CRT dataset. This work represents the first evaluation of the software on an clinically accepted ECG database [5].

\section{Method and software description}

The VED Meter is a stand-alone desktop application that includes rich graphic user interface. Although the computation is fully automatic, the user can adjust computational settings. We describe the various processing steps of the ECG signals and illustrate these steps in the flowchart in Figure 1.

\subsection{Software and hardware requirements}

The VED Meter requires the Windows ${ }^{\mathrm{TM}}$ version 7 operating system or higher. The application is written in C\# programming language and uses .NET framework 4.5. Application requires at least $250 \mathrm{MB}$ of free RAM and 1 MB of HDD space. Because the processing is heavily parallelized, it strongly benefits from higher number of available CPU cores. Average computing time of 10minutes long ECG record on CPU i7-5600U (2cores, 4 threads) is 14.7 seconds.

\subsection{Signal Input}

We applied the proposed tool to the ECGs acquired during the MADIT-CRT trial recorded at baseline pre implantation. These body-surface ECG were recorded using the Mason-Likar lead configurations, i.e., 12-lead ECG recording, and with a sampling frequency of $1 \mathrm{kHz}$, and an amplitude resolution of 16-bit $( \pm 5 \mathrm{mV})$. The ECG signals were stored in ISHNE format [6] and included beat annotations stored in a separate file. Ten minutes of resting supine position were analysed. 


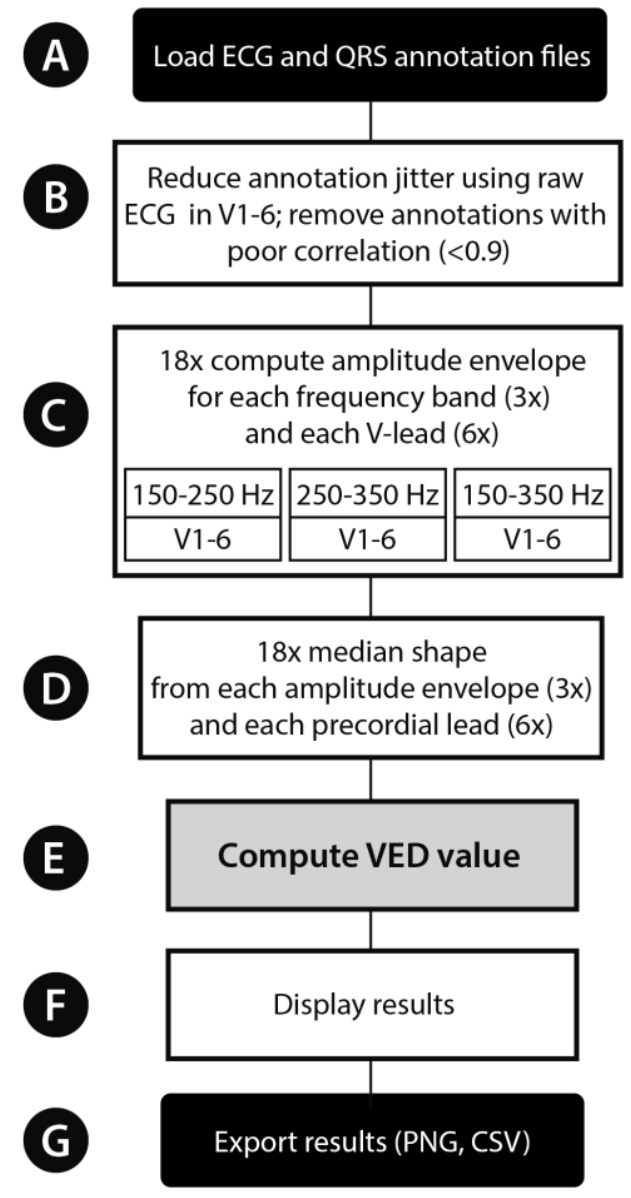

Figure 1: Processing flowchart. Data are loaded from ISHNE ECG file (12-lead, $1 \mathrm{kHz}, 16-\mathrm{bit})$ and from QRS annotation file (A); annotation jitter is reduced using raw ECG signal in V1-V6 (B); amplitude envelopes are computed (C); 18 average median shapes are assessed from amplitude envelopes (D); resultant VED value is computed (E) and displayed as well as partial results (F). These results may be exported into image or table $(\mathrm{G})$.

\subsection{QRS annotations}

By default, VED Meter uses only cardiac beats associated with sinus rhythm. Non-sinus beats are filtered out and an average QRS shape is built from the set of normal beats. QRS annotation jitter is reduced by using Pearson correlation in all six precordial leads. Finally, QRS complexes which do not correlate enough to the averaged QRS shape $(r<0.9)$ are excluded from further processing.

\subsection{ECG signal transformation}

Signals from precordial leads V1 to V6 are transformed into amplitude envelopes in three frequency ranges (150-
250, 250-350, and 150-350 Hz) using a fast Fourier and Hilbert transforms (Fig.2 A, B). Ultimately, a total of 18 amplitude envelopes are obtained from which signal averaging is applied.

\subsection{Signal averaging using medians}

Due to the low signal-to-noise ratio of amplitude envelopes the signal averaging of QRS complexes is used. And for greater resistance to artefacts, median function is used instead of the computation of mean.

Data for signal averaging are extracted from a $350 \mathrm{~ms}$ window centred on the annotation marks (Fig. 2 B). Median shape (Fig.2 C) is built for each precordial lead V1-V6. The baseline is corrected using subtraction of mean voltage from a window (100 ms long) starting 200 $\mathrm{ms}$ after the QRS annotation mark. This is processed for the three frequency bands generating 18 median shapes. For each of them the following features are computed: time position of maxima $m$ (Fig.2 $\mathrm{C}-\mathrm{m}$ ), position of center of gravity $c($ Fig.2 $\mathrm{C}-\mathrm{c})$ and their average $a($ Fig.2 $\mathrm{C}-\mathrm{a})$.

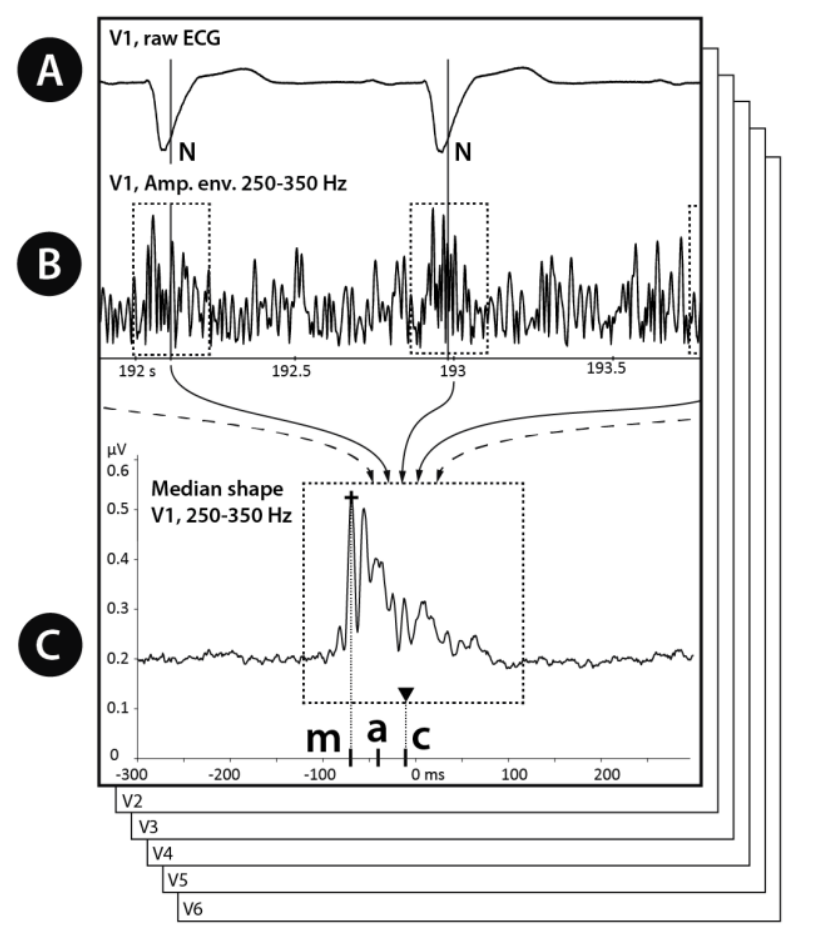

Figure 2: Computation of the signal averaging for lead V1 in frequency range $250-350 \mathrm{~Hz}$. Raw ECG signal (A) is transformed into amplitude envelope (B); QRS annotation marks with corrected jitter $(\mathrm{N})$ serve as a trigger during assessing of median shape (C). Source features of each median shape are time position of maxima (m), center of mass (c) and their average (a). This process is applied to each chest lead and each frequency band. 


\subsection{Computation of the VED}

First, the sum $S_{F}$ for each frequency band is derived from the median shapes extracted from V1-V6:

$$
\begin{gathered}
S_{F}=d m_{16 F}+d c_{16 F}+d a_{16 F}+d m_{\text {all } F} \\
+d c_{\text {all } F}+d a_{\text {all } F}
\end{gathered}
$$

where $d m_{16}, d c_{16}$ and $d a_{16}$ are time differences between points $m, c$ and $a$ respectively (Fig. 2, $m=$ maximum, $c=$ center of gravity, $a=$ mean between $m$ and $c$ ) in leads $\mathrm{V} 1$ and $\mathrm{V} 6 ; d m_{\text {all }}, d c_{\text {all }}$ and $d a_{\text {all }}$ are maximal time difference among points $m, c$ and $a$ across all precordial leads. Index $F$ signalizes used frequency band (150-250, 250-350 and 150-350 Hz).

Finally, the value $V E D$ is defined as the average:

$$
V E D=\frac{S_{150-250}+S_{250-350}+S_{150-350}}{18}
$$

where $S$ are sums derived from specific frequency ranges.

\section{Results}

The Figure 3 is the snapshot of the VED Meter user interface delivering the VED value (Fig 3- D). Importantly, the software enables the visual review of the signals used to compute the VED value (Fig.3 - A) by displaying the averaged QRS and median shapes (Fig.3 C). The set of used annotation (jitter-corrected) marks may be checked and exported to the SignalPlant [7] annotation *.sel file for further analysis and inspection.

\subsection{VED values and ventricular conduction defects}

We compared (Fig. 4) the values of VED for the various type of ventricular conduction abnormalities, i.e., left $(n=663)$ and right $(n=109)$ bundle branch blocks (LBBB, and RBBB, respectively) and intra-ventricular conduction defects (IVCD, $\mathrm{n}=159$ ). Median VED value/ interquartile range in milliseconds was 51.4/ 33.5 (LBBB), -30.0/ 27.1 (RBBB) and 16.9/ 19.4 (ICVD). Nonparametric Kruskal-Wallis test shown statistical difference $(\mathrm{p}<0.001)$ across all 3 groups.

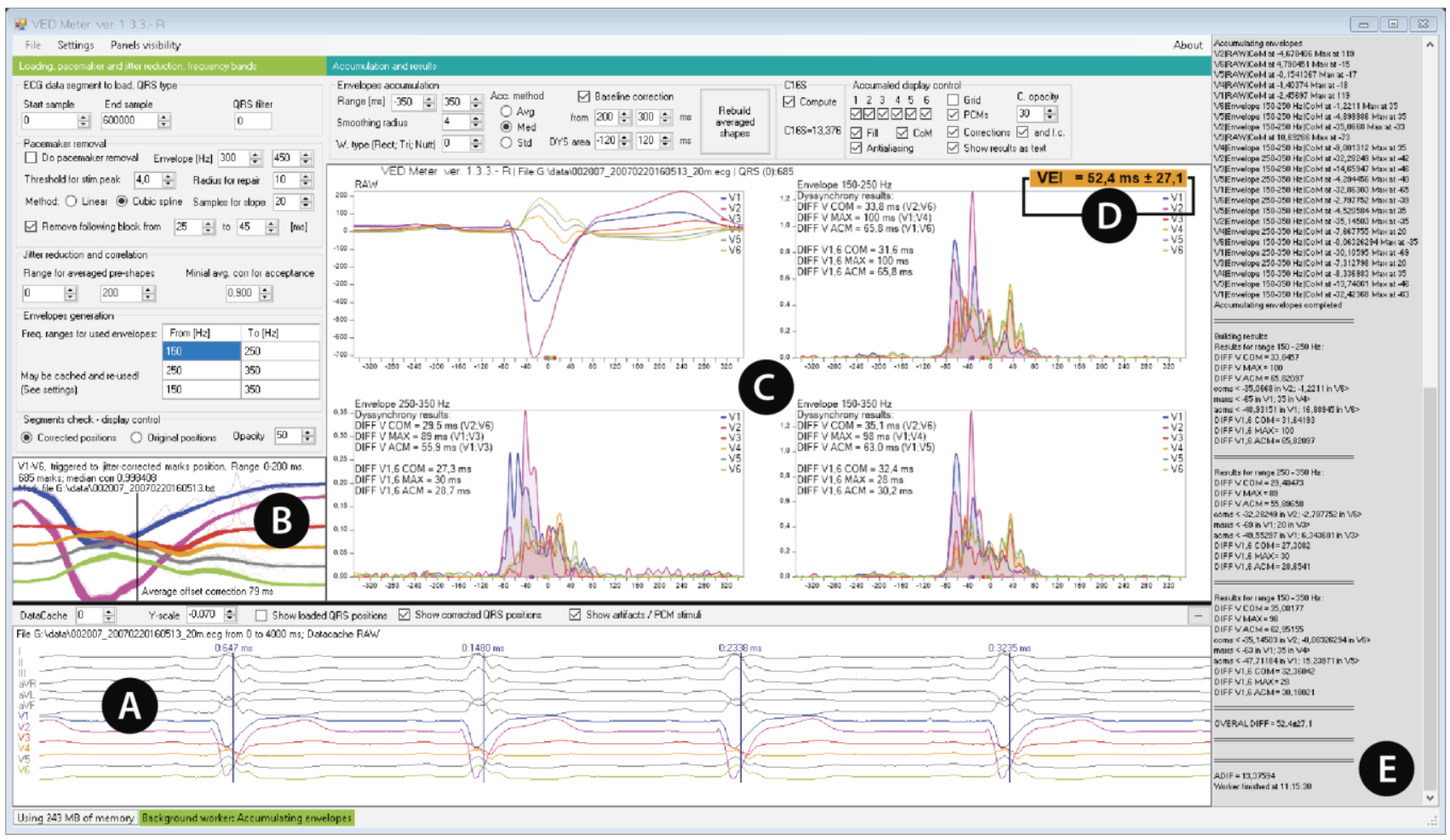

Figure 3: VED Meter - GUI and results display. Interactive preview of input signal (A), panel allowing check of jitter removal (B), display of averaged QRS, median shapes and intermediate results (C) and the VED value (D). Additional computing info is displayed in the right panel (E). 


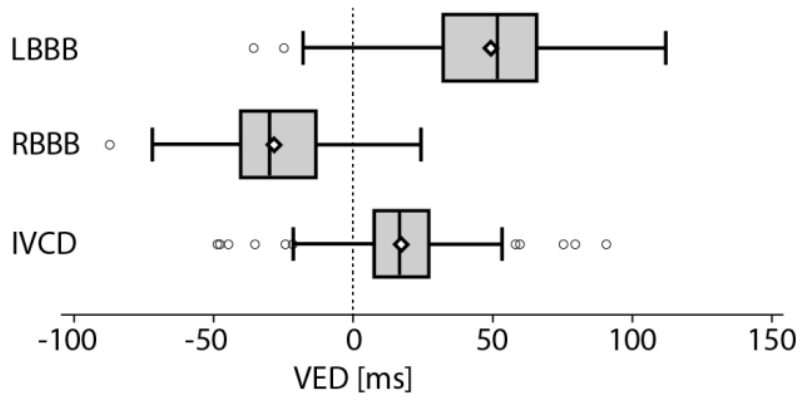

Figure 4: Distribution of VED value in MADIT-CRT trial for LBBB $(n=663)$, RBBB $(n=109)$, and IVCD $(n=159)$ patients measured at the baseline. Kruskal-Wallis test shows $\mathrm{p}<0.001$.

\section{Discussion}

The VED Meter produces VED values associated with ventricular electrical dyssynchrony in heart failure patients. The processing is fully automatic (may run in batch) without any need of manual adjudication.

Measuring the level of electrical dyssynchrony and initially the type of conduction abnormalities is crucial since clinical studies have shown that CRT is beneficial primarily in patients with LBBB. The VED provide a value that filter out patient with RBBB if only those with positive values are retained. The next step is to study the association between the VED value and the patient outcome after implantation. Our hypothesis is that the higher the level of VED, the more beneficial is the CRT outcome.

We plan to adopt existing QRS detection mechanism and morphology clustering from the SignalPlant software [7] and to develop more reliable elimination of pacemaker stimuli from ECG signal. All this functionality should be included in the public version of VED Meter, allowing processing of non-annotated ECG data. Moreover, the option for processing ECG signals with an active pacemaker may allow for optimizing CRT settings in regard to resultant ventricle dyssynchrony.

\section{Conclusion}

We presented a software tool, VED Meter, for the fully automatic computing of electrical ventricular delay. VED Meter software was used to analyze data from the MADIT-CRT trial. The resulting VED parameter (in ms) produces conduction-specific values which reflect delayed activation of the septum and the right ventricle (negative values) or delayed activation of the left ventricle lateral wall (positive values). Based on our results, we conclude that the VED Meter may be considered as a new tool to measure conduction abnormalities connected to the electrical dyssynchrony.

\section{Acknowledgements}

The research was supported by Czech Academy of Sciences project MSM 100651602, the Czech Science Foundation project no. 17-13830S, by MEYS CR projects (no. LO1212 and no. LQ1605) and by CAS (no. RVO:68081731).

\section{References}

[1] N. Varma, M. Manne, D. Nguyen, J. He, M. Niebauer, and P. Tchou, "Probability and magnitude of response to cardiac resynchronization therapy according to QRS duration and gender in nonischemic cardiomyopathy and LBBB," Hear. Rhythm, vol. 11, no. 7, pp. 11391147, 2014.

[2] M. Dupont, J. Rickard, B. Baranowski, N. Varma, T. Dresing, A. Gabi, M. Finucan, W. Mullens, B. L. Wilkoff, and W. H. W. Tang, "Differential response to cardiac resynchronization therapy and clinical outcomes according to QRS morphology and QRS duration," J. Am. Coll. Cardiol., vol. 60, no. 7, pp. 592-598, 2012.

[3] P. Jurak, J. Halamek, F. Plesinger, T. Reichlova, J. Lipoldova, M. Novak, K. Jurakova, and P. Leinveber, "An additional marker of ventricular dyssynchrony," in 2015 Computing in Cardiology Conference (CinC), 2015, pp. 77-80.

[4] P. Jurak, J. Halamek, J. Meluzin, F. Plesinger, T. Postranecka, J. Lipoldova, M. Novak, V. Vondra, I. Viscor, L. Soukup, P. Klimes, P. Vesely, J. Sumbera, K. Zeman, R. S. Asirvatham, J. Tri, S. J. Asirvatham, and P. Leinveber, "Ventricular dyssynchrony assessment using ultra-high frequency ECG technique," J. Interv. Card. Electrophysiol., vol. 49, no. 3, pp. 245-254, Sep. 2017.

[5] A. J. Moss, M. W. Brown, D. S. Cannom, J. P. Daubert, M. Estes, E. Foster, H. M. Greenberg, W. J. Hall, D. Ph, S. L. Higgins, H. Klein, M. Pfeffer, D. Wilber, and W. Zareba, "Trial - Cardiac Resynchronization Therapy ( MADIT-CRT ): Design and Clinical Protocol," Ann. noninvasive Electrocardiol., vol. 10, no. 4, pp. 34-43, 2005.

[6] F. Badilini, "The ISHNE Holter standard output file format," Annals of Noninvasive Electrocardiology, vol. 3, no. 3 I. pp. 263-266, 1998.

[7] F. Plesinger, J. Jurco, J. Halamek, and P. Jurak, "SignalPlant: an open signal processing software platform," Physiol. Meas., vol. 37, no. 7, pp. N38-N48, 2016.

Address for correspondence:

Filip Plesinger

Institute of Scientific Instruments of the Czech Academy of Sciences

Kralovopolska 147

Brno 61264

Czech Republic

E-mail address: fplesinger@isibrno.cz 